\title{
Excited-State Dynamics of Nitroperylene in Solution: Solvent and Excitation Wavelength Dependence
}

\author{
Omar F. Mohammed ${ }^{\dagger}$ and Eric Vauthey* \\ Department of Physical Chemistry, University of Geneva, 30 quai Ernest-Ansermet, \\ CH-1211 Geneva 4, Switzerland \\ Received: January 11, 2008; In Final Form: February 12, 2008
}

\begin{abstract}
The photophysics and excited-state dynamics of nitroperylene (NPe) in solvents of various polarities and viscosities, including a room-temperature ionic liquid, have been investigated by femtosecond-resolved transient absorption spectroscopy. The excited-state absorption spectrum was found to depend substantially on solvent polarity. In the most polar solvents, it is very similar to that of the NPe radical cation generated upon bimolecular quenching by an electron acceptor, denoting a substantial charge-transfer character of the $\mathrm{S}_{1}$ state. Contrary to smaller nitroaromatic compounds, NPe in the $S_{1}$ state does not undergo ultrafast intersystem crossing (ISC) but decays mainly by internal conversion (IC). In nonprotic solvents, IC involves low-frequency modes with large amplitude motion associated with the nitro group and depends on both the solvent viscosity and polarity. It takes place on a $100 \mathrm{ps}$ time scale in acetonitrile, while in cyclohexane, it is slow enough for ISC to become competitive. Moreover, both the fluorescence quantum yield and the excited-state dynamics were found to differ, depending on which side of the $S_{0}-S_{1}$ absorption band excitation was performed. This dependence is explained by the inhomogeneous nature of the absorption spectrum arising from a distribution of twist angles of the nitro group relative to the aromatic plane. On the other hand, such excitation wavelength effects were not observed in protic solvents, where the excited-state lifetime was found to be substantially shorter than that in nonprotic solvents. This behavior is rationalized in terms of a H-bonding interaction, which limits the torsional disorder of NPe and favors ultrafast nonradiative deactivation of the excited state. Transient absorption measurements performed for comparative purpose with nitropyrene in acetonitrile confirm the occurrence of ultrafast ISC in smaller nitroaromatic compounds.
\end{abstract}

\section{Introduction}

Addition of a nitro group to an aromatic hydrocarbon is known to considerably affect its photophysical and photochemical properties. Indeed, benzene, stilbene, and polycyclic aromatic hydrocarbons such as naphthalene, anthracene, and pyrene are no longer fluorescent when nitro-substituted. Two phenomena can account for this effect, the enhancement of intersystem crossing (ISC) and that of internal conversion (IC). The presence of $\mathrm{n} \pi^{*}$ states associated with the nitro group enables ultrafast ISC between $\pi \pi^{*}$ and $n \pi^{*}$ states of the singlet and triplet manifolds. ${ }^{1}$ Transient absorption measurements on several nitroaromatic compounds have revealed a triplet population buildup time of a few picoseconds. ${ }^{2-5}$ Because of this efficient ISC and thus large triplet yield, the photochemistry of most nitroaromatic compounds essentially takes place from the $T_{1}$ state. ${ }^{6-10}$

More recently, the fluorescence decays of a series of polycyclic nitroaromatic hydrocarbons such as 1-nitronaphthalene, 9-nitroanthracene, and 1-nitropyrene in methanol have been shown to be ultrafast, with average time constants on the subpicosecond time scale. ${ }^{11,12}$ These very small lifetimes have been ascribed to highly efficient ISC, and as a result, these compounds can be considered as the organic molecules with the fastest ISC. It should nevertheless be noted that despite the

\footnotetext{
* To whom correspondence should be addressed. E-mail: eric.vauthey@ chiphy.unige.ch.

Present address: RIKEN (The Institute of Chemical and Physical
} Research), 2-1 Hirosawa, Wako, Saitama 351-0198, Japan. fact that the fluorescence dynamics was measured with high precision, transient absorption measurements that would confirm population of the triplet state were not carried out.

The presence of a nitro group has also been shown to lead to ultrafast IC. For example, Ernsting and co-workers have reported that para-nitroaniline in water undergoes $S_{1}-S_{0}$ IC in $250 \mathrm{fs}$ and that this process is coupled to the torsion of the $\mathrm{NO}_{2}$ group relative to the aromatic ring, which brings the excited-state population toward a conical intersection with the ground state. ${ }^{13,14}$ Similarly, the unusually fast $\mathrm{T}_{1}-\mathrm{S}_{0}$ ISC in nitrobenzene has been ascribed to the substantial geometry differences between these two states at the $\mathrm{NO}_{2}$ group. ${ }^{15}$

Quantum chemistry calculations at different levels of theory on nitrobenzene and para-nitroaniline in vacuum indicate that the electronic ground-state potential along the twist coordinate of the nitro group is very shallow. ${ }^{14,16}$ As a consequence, a wide distribution of $\mathrm{NO}_{2}$-twist angles is predicted at room temperature. As the curvature of the excited-state potential along this coordinate differs, photoselection of molecules with a relatively narrower distribution of twist angles is, in principle, possible. Consequently, the excited-state dynamics can be expected to depend on the excitation wavelength.

We report here on a detailed investigation of the photophysics and excited-state dynamics of 3-nitroperylene (NPe) in solvents of varying polarity and viscosity, including a room-temperature ionic liquid, using femtosecond transient absorption spectroscopy. NPe has been chosen for mainly three reasons. First, because of the relatively large size of its aromatic system, the 

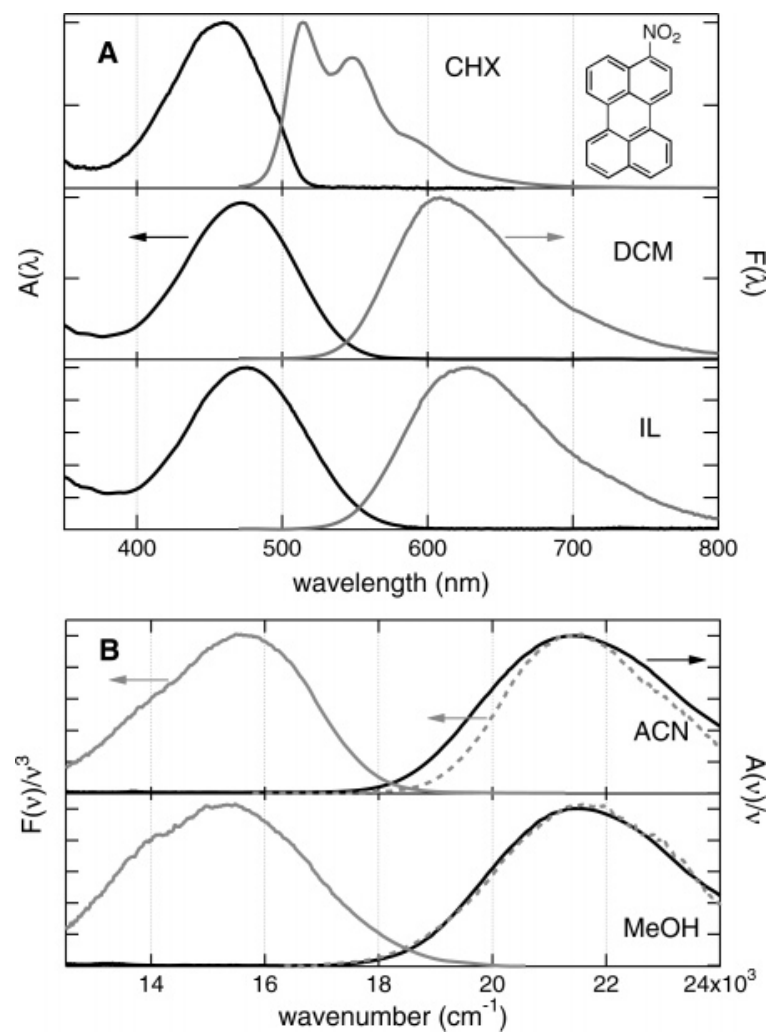

Figure 1. Absorption and fluorescence spectra (440 nm excitation) of $\mathrm{NPe}$ in (A) CHX, DCM, and IL in wavelength units and (B) in ACN and $\mathrm{MeOH}$ in wavenumber (the dotted lines represent the emission spectra as a function of a transformed frequency $\tilde{v}_{\mathrm{fl}}^{\prime}$; see text).

lowest triplet $n \pi^{*}$ state of NPe might be expected to lie above the $S_{1} \pi \pi^{*}$ state. In this case, ISC should no longer be favored by spin-orbit coupling and be thus inefficient. Consequently, NPe should have totally different photophysics than the smaller nitro-aromatic hydrocarbons investigated so far. Second, transient absorption measurements of NPe should give information on the effect of partial electron withdraw on the excited-state absorption spectrum of the perylene (Pe) moiety. Pe is very often used as a donor in photoinduced ET processes. ${ }^{17-20}$ In such reactions, the transient absorption spectrum changes from that of $\mathrm{Pe}^{*}\left(\mathrm{~S}_{1}\right)$ with a band at $700 \mathrm{~nm}$ to that of the cation $\mathrm{Pe}^{\bullet+}$ with a band at $540 \mathrm{~nm}$. If the primary ET product is a strongly coupled ion pair, the net positive charge on $\mathrm{Pe}^{\bullet+}$ should be inferior to one, and the absorption spectrum of the paired $\mathrm{Pe}^{\bullet+}$ should differ from that of the free $\mathrm{Pe}^{\bullet+}$. However, such a difference has never been reported, so far. Because of the electron-withdrawing property of the $\mathrm{NO}_{2}$ substituent, a partial charge transfer from the Pe to the nitro moieties can be anticipated in the $S_{1}$ state. Thus, transient absorption measurements with NPe should reveal how the spectrum of Pe with only a partial positive charge differs from those of $\mathrm{Pe} *\left(\mathrm{~S}_{1}\right)$ and $\mathrm{Pe}^{\bullet+}$. Third, the location of the $\mathrm{S}_{0}-\mathrm{S}_{1}$ absorption band of NPe is particularly well suited for investigating the effect of excitation wavelength on the excited-state dynamics.

Transient absorption measurements with nitropyrene in acetonitrile will additionally be presented for comparative purpose.

\section{Experimental Section}

Samples. 3-Nitroperylene (NPe) was synthesized according to the procedure described in ref 21 and was purified by column chromatography. 1-Nitropyrene (NPy, Aldrich) was used as received, while tetracyanoethylene (TCNE, Aldrich) was sub-
TABLE 1: Absorption, $\lambda_{\mathrm{abs}}$, and Fluorescence Maxima, $\lambda_{\mathrm{fl}}$, and Fluorescence Quantum Yields, $\Phi_{\mathrm{fl}}$, at Two Different Excitation Wavelengths

\begin{tabular}{lcccc}
\hline solvent & $\lambda_{\text {abs }}(\mathrm{nm})$ & $\lambda_{\mathrm{fl}}(\mathrm{nm})$ & $\Phi_{\mathrm{fl}}(400 \mathrm{~nm})$ & $\Phi_{\mathrm{fl}}(495 \mathrm{~nm})$ \\
\hline $\mathrm{CHX}$ & 460 & 514 & 0.15 & 0.19 \\
$\mathrm{BCl}$ & 475 & 576 & & \\
$\mathrm{CHCl}$ & 471 & 607 & & \\
$\mathrm{DCM}$ & 473 & 698 & 0.026 & 0.047 \\
$\mathrm{MeOH}$ & 462 & 619 & 0.002 & 0.002 \\
$\mathrm{DMF}$ & 472 & 615 & & \\
$\mathrm{ACN}$ & 464 & 608 & 0.010 & 0.012 \\
$\mathrm{DMSO}$ & 475 & 625 & 0.032 & 0.053 \\
$\mathrm{NMF}$ & 472 & 645 & 0.004 & 0.004 \\
$\mathrm{IL}$ & 476 & 627 & &
\end{tabular}

limed. The solvents, cyclohexane (CHX), chlorobenzene ( $\mathrm{BCl})$, chloroform $\left(\mathrm{CHCl}_{3}\right)$, dichloromethane (DCM), acetonitrile (ACN), methanol (MeOH), $N$-methylformamide (NMF), and dimethylsulfoxide (DMSO), were from Fluka, the ionic liquid (IL), 1-ethyl-3-methylimidazolium-ethylsulfate, was from Solvent Innovation $\mathrm{GmbH}$, and perdeuterated methanol (MeOD) was from Armar Chemicals AG. All solvents were used without further purification.

Steady-State Measurements. Absorption spectra were recorded on a Cary 50 spectrophotometer, while fluorescence spectra were measured on a Cary Eclipse fluorimeter. The fluorescence spectra were corrected for the wavelength-dependent sensitivity of the detection. Quantum yield measurements were performed against coumarin $153 .{ }^{22}$

Time-Resolved Fluorescence. Fluorescence lifetime measurements were carried out with the time-correlated singlephoton counting (TCSPC) technique as described elsewhere. ${ }^{23}$ Excitation was performed at a repetition rate of $10 \mathrm{MHz}$ with $\sim 60$ ps pulses generated with a laser diode at $395 \mathrm{~nm}$ (Picoquant models LDH-P-C-400B). The full width at half-maximum (FWHM) of the instrument response function (IRF) was around 200 ps. The absorbance of the sample solutions was adjusted to have an optical density at the excitation wavelength of 0.1 on $1 \mathrm{~cm}$.

Transient Absorption (TA). The experimental setup has been described in detail earlier. ${ }^{24}$ Excitation was performed either at $400 \mathrm{~nm}$ using the frequency-doubled output of a standard $1 \mathrm{kHz}$ amplified Ti:Sapphire system (Spectra-Physics) or at $495 \mathrm{~nm}$ with a home-built two-stages non-collinear optical parametric amplifier. ${ }^{25}$ The energy per pulse at the sample was around 5 and $1-2 \mu \mathrm{J}$ at $400 \mathrm{~nm}$ and at $495 \mathrm{~nm}$, respectively. The polarization of the probe pulses was at magic angle relative to that of the pump pulses. All spectra were corrected for the chirp of the white light probe pulses. The FWHM of the IRF was $\sim 200 \mathrm{fs}$. The sample solutions were kept in a $1 \mathrm{~mm}$ thick quartz cell and were continuously stirred by $\mathrm{N}_{2}$ bubbling. The absorbance of NPe was adjusted to have an optical density of $0.05-0.1$ at $400 \mathrm{~nm}$ and $0.2-0.3$ at $495 \mathrm{~nm}$.

Quantum Chemistry Calculations. Ground-state gas-phase geometry optimization was performed at the density functional level of theory (DFT) using the B3LYP functional ${ }^{26}$ and a [3s 2 p1d] basis set. ${ }^{27}$ Electronic vertical excitation energies and excited-state geometries were computed with time-dependent density functional theory (TDDFT) using the same functional and basis set. ${ }^{28}$ The calculations were carried out using Turbomole version 5.9. ${ }^{29,30}$

\section{Results}

Steady-State Spectroscopy. Absorption and fluorescence spectra of NPe in several solvents are shown in Figure 1A. The absorption spectrum above $350 \mathrm{~nm}$ depends only weakly on 


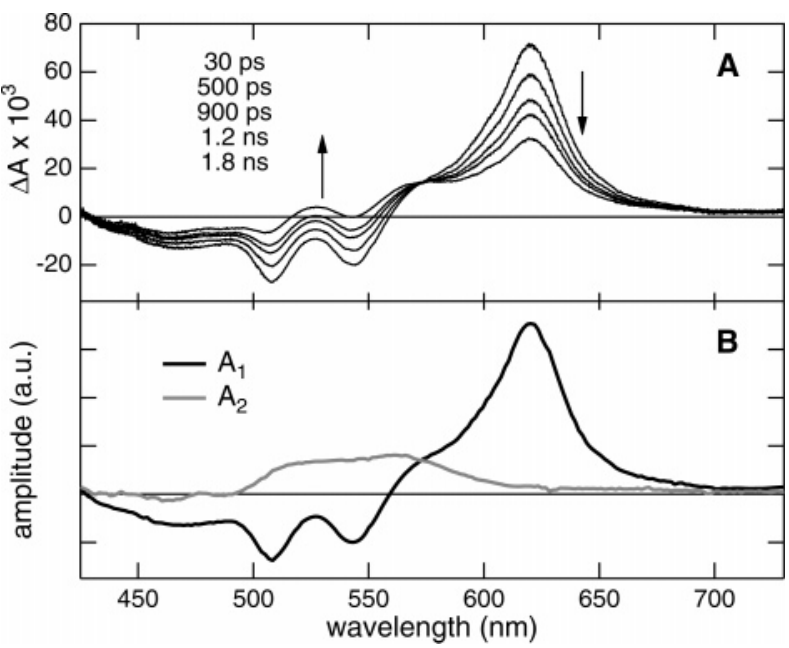

Figure 2. (A) TA spectra recorded at different time delays after 400 $\mathrm{nm}$ excitation of NPe in CHX; (B) spectral dependence of the amplitude factors obtained from global analysis (see text).

the solvent and consists of a broad and structureless band peaking between $460 \mathrm{~nm}$ in CHX and $476 \mathrm{~nm}$ in IL (see Table 1). This band differs substantially from that of the parent compound $\mathrm{Pe}$, which exhibits a pronounced vibrational progression with the lowest-energy maximum at $436 \mathrm{~nm}$ in CHX. In solvents of similar refractive index, $n$, the absorption band maximum, in energy units, decreases linearly with the polarity function, $f\left(\epsilon_{\mathrm{s}}\right)=\left(2 \epsilon_{\mathrm{s}}-1\right) / 2\left(\epsilon_{\mathrm{s}}+1\right)$, where $\epsilon_{\mathrm{s}}$ is the static dielectric constant. Similarly, in solvents of similar dielectric constant, the absorption band maximum decreases linearly with $f\left(n^{2}\right)=\left(2 n^{2}-1\right) / 2\left(n^{2}+1\right)$. Dipole-dipole, dipole-dipoleinduced, and dispersion interactions thus contribute to the solvatochromism of the $\mathrm{S}_{0}-\mathrm{S}_{1}$ absorption band of NPe. ${ }^{31,32}$

The fluorescence spectrum of NPe exhibits a larger solvent dependence. First, in CHX, it presents a vibrational progression, although not as pronounced as that of Pe, while it is broad and structureless in the other solvents. Second, it displays a substantial solvatochromism, which is dominated by the dipoledipole interaction. Indeed a plot of the Stokes shift, $\tilde{v}_{\text {abs }}^{\max }-$ $\tilde{v}_{\mathrm{fl}}^{\max }$ versus $f\left(\epsilon_{\mathrm{s}}\right)-f\left(n^{2}\right)$ is linear with a $5120 \mathrm{~cm}^{-1}$ slope. Inserting this value in the Lippert-Mataga relationship, ${ }^{33,34}$ with a cavity radius of $3.9 \AA$, results in an increase of the permanent dipole moment from the ground to excited state of 7.7 D. The dielectric constant of IL is not known, but according to the spectra measured in this solvent, its effective polarity is similar to that of DMSO.

At first sight, the mirror image relationship between absorption and emission bands seems to hold in all solvents except CHX. However, a careful examination of the spectra in wavenumber units and after correct representation ${ }^{35}$ reveals that such mirror symmetry is realized in protic solvents but not in the other solvents. In nonprotic solvents, the absorption band is noticeably broader than the fluorescence band. This is illustrated in Figure 1B, which displays the fluorescence spectra of $\mathrm{NPe}$ in $\mathrm{ACN}$ and in $\mathrm{MeOH}$ as a function of a transformed frequency axis $\tilde{v}_{\mathrm{fl}}{ }^{\prime}=2 \tilde{v}_{0}-\tilde{v}_{\mathrm{fl}}$, where $\tilde{v}_{0}=\left(\tilde{v}_{\mathrm{abs}}^{\max }+\tilde{v}_{\mathrm{fl}}^{\max }\right) / 2$.

Finally, the fluorescence quantum yield, $\Phi_{\mathrm{fl}}$, upon $400 \mathrm{~nm}$ excitation also depends substantially on the solvent, as shown in Table 1. It is as large as 0.15 in CHX and drops abruptly below 0.05 as the solvent polarity increases, the lowest values being found in the protic solvents. In nonprotic solvents, the fluorescence quantum yield depends on the excitation wavelength and is larger upon red side (495 $\mathrm{nm}$ ) than upon blue side $(400 \mathrm{~nm})$ excitation. The difference is quite modest in $\mathrm{ACN}$

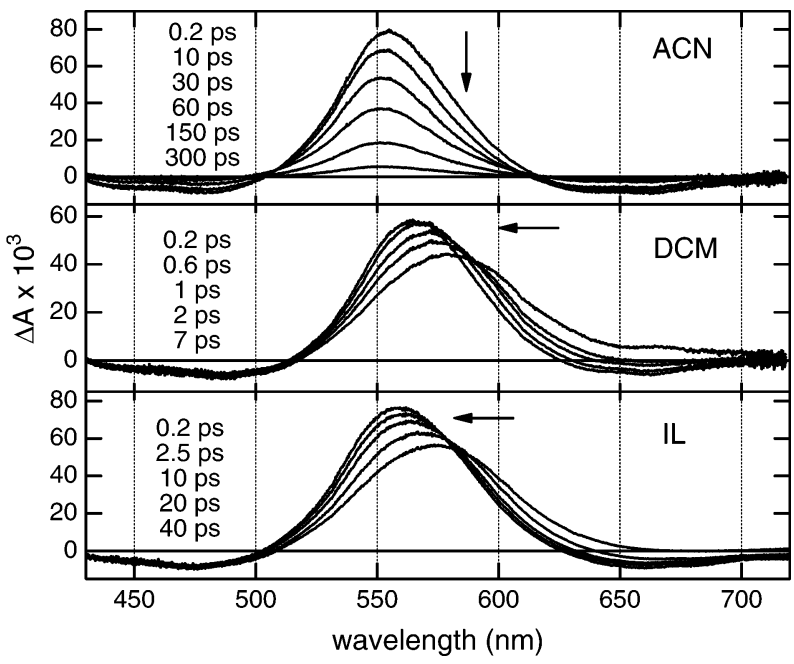

Figure 3. TA spectra recorded with NPe at different time delays after $400 \mathrm{~nm}$ excitation.

and CHX but is pronounced in DMSO and DCM, where the quantum yield increases by more than $50 \%$.

Time-Resolved Spectroscopy. TA spectra recorded with NPe in CHX upon $400 \mathrm{~nm}$ excitation are depicted in Figure 2A. They consist of a negative band located between 420 and $560 \mathrm{~nm}$ that can be ascribed to both the bleach of the $S_{0}-S_{1}$ absorption and stimulated $\mathrm{S}_{1}-\mathrm{S}_{0}$ emission and in a positive band centered at $630 \mathrm{~nm}$ that can be assigned to $S_{1}-S_{n}$ absorption. Apart from a partial $\sim 25$ ps rise of the signal intensity in the stimulated emission and the positive TA band regions, the spectra show little evolution over the first few hundreds of picoseconds. The TA intensity of both the positive and negative bands decreases on the nanosecond time scale, while a new absorption feature appears between about 500 and $600 \mathrm{~nm}$.

The TA spectra recorded after 30 ps have been analyzed globally with the following function that assumes a reaction scheme where population is transferred with a time constant $\tau$ from the $S_{1}$ state to a second state, which is responsible for the absorption feature between 500 and $600 \mathrm{~nm}$

$$
f(\lambda, t)=A_{1}(\lambda) \exp (-t / \tau)+A_{2}(\lambda)[1-\exp (-t / \tau)]
$$

This function could be very well fitted to the TA time profiles at all wavelengths, and a best-fit value of $1.8 \mathrm{~ns}$ was obtained for $\tau$. The spectra of the amplitude factors, $A_{1}$ and $A_{2}$, are depicted in Figure 2B. The spectrum of $A_{1}$ is very similar to the early TA spectrum and can be ascribed to the $S_{1}$-state population and to the depleted ground-state population. On the other hand, the spectrum of $A_{2}$ is only positive and peaks at around $560 \mathrm{~nm}$. Given that NPe in CHX does not undergo chemical reaction, this long-lived band can be safely ascribed to the lowest triplet excited state of NPe. As the TA setup did not allow time delays longer than about $2 \mathrm{~ns}$ to be investigated, the fluorescence decay of NPe in CHX was also measured by TCSPC in order to have a more reliable excited-state lifetime. The time profile of the fluorescence intensity at $560 \mathrm{~nm}$ could be very well reproduced using an exponential function with a 1.70 ns time constant, in good agreement with the global analysis result.

As shown in Figure 3, the TA spectra of NPe in polar solvents differ considerably from those in CHX. They consist of an intense positive band centered between 550 and $600 \mathrm{~nm}$, depending on the solvent and time, with two negative features at both the blue and red sides. Comparison with the steadystate spectra indicates that the blue negative band is mostly due 


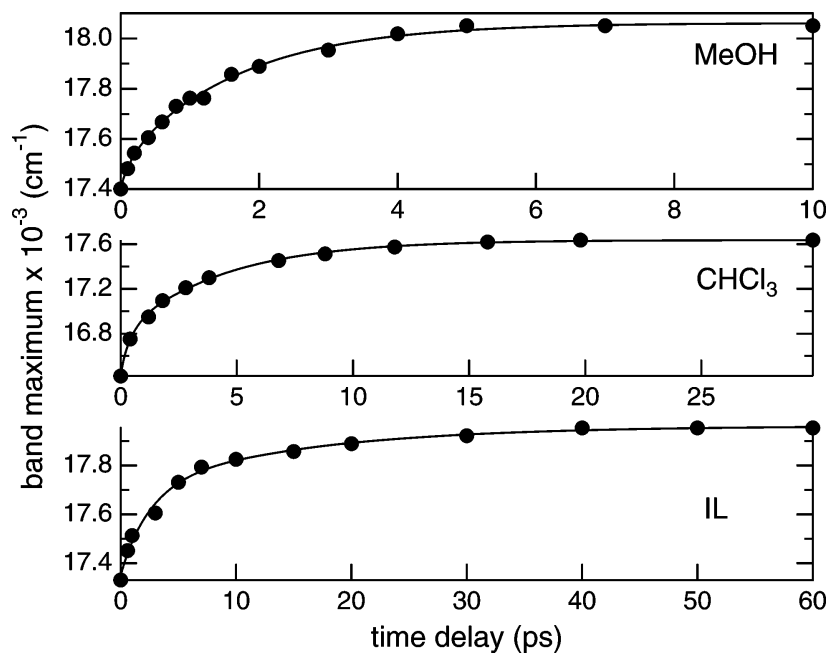

Figure 4. Time dependence of the positive TA band maximum and the best biexponential fit.

TABLE 2: Initial, $\lambda_{\mathrm{i}}$, and Final, $\lambda_{\mathrm{f}}$, Wavelength of the Positive TA Band Maximum and Time Constants, $\tau_{\mathrm{s}, \mathrm{i}}$, (with Relative Amplitudes) Obtained from the Biexponential Fit of Its Time Dependence

\begin{tabular}{llll}
\hline solvent & $\lambda_{\mathrm{i}}-\lambda_{\mathrm{f}}(\mathrm{nm})$ & \multicolumn{1}{c}{$\tau_{\mathrm{s}, 1}(\mathrm{ps})$} & $\tau_{\mathrm{s}, 2}(\mathrm{ps})$ \\
\hline $\mathrm{CHCl}_{3}$ & $609-567$ & $0.38(0.3)$ & $4.3(0.7)$ \\
$\mathrm{DCM}$ & $579-565$ & $0.19(0.4)$ & $1.47(0.6)$ \\
$\mathrm{MeOH}$ & $575-554$ & $0.15(0.15)$ & $1.7(0.85)$ \\
$\mathrm{ACN}$ & $556-554$ & & \\
$\mathrm{DMSO}$ & $587-555$ & $1.2(0.55)$ & $3.1(0.45)$ \\
$\mathrm{NMF}$ & $568-555$ & $0.9(0.5)$ & $5.6(0.5)$ \\
$\mathrm{IL}$ & $574-559$ & $2.4(0.55)$ & $14.9(0.45)$
\end{tabular}

to the bleach of the ground-state absorption, while the other band originates from stimulated emission. However, the latter also contributes to the TA in the region where the signal is positive. In ACN, the spectral shape is essentially independent of time, and the TA intensity over the whole spectral window decays to zero within about 400 ps. In the other solvents, a substantial spectral dynamics is observed during the first few tens of picoseconds after excitation (Figure 3). In DCM, for example, the positive band peaks first at $579 \mathrm{~nm}$ and then increases and shifts to $565 \mathrm{~nm}$. An even more pronounced effect is observed in $\mathrm{CHCl}_{3}$ as the initial position of the band is more red-shifted. The initial and final peak positions in all solvents investigated are listed in Table 2 . The temporal variation of the peak position in a few solvents is depicted in Figure 4. The solid lines are the best fits of a biexponential function, whose time constants and amplitudes are listed in Table 2. The time constants found agree well with those reported for the dynamic Stokes shift of coumarin 153 in the same solvents. ${ }^{36}$ Consequently, the spectral shift of the positive TA band can be safely ascribed to solvent relaxation by diffusive motion. Solvation is also known to partially take place upon inertial solvent motion. ${ }^{37}$ However, this process occurs with a $\sim 100$ fs time constant and is too fast to be totally resolved here. ${ }^{38}$ On the other hand, the spectral shift in ACN is too small to allow a time constant to be extracted. The time constants obtained in IL seem to correspond to the fastest components of solvation. Indeed, dynamic Stokes shifts measurements performed in ionic liquids reveal the presence of slower solvation components on the subnanosecond time scale. ${ }^{39-41}$ This slower part of solvation is most probably not visible here because of the relatively short excited-state lifetime. Apart from CHX, the TA bands in all solvents investigated decay to zero within the time window of

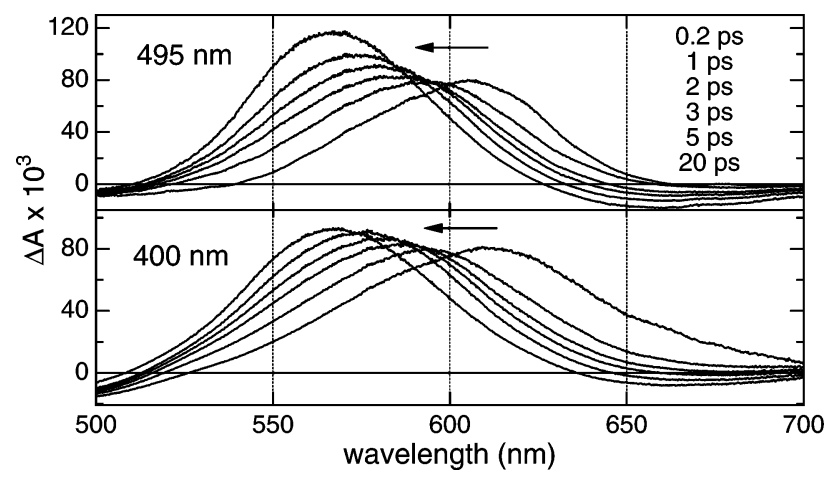

Figure 5. TA spectra recorded with $\mathrm{NPe}$ in $\mathrm{CHCl}_{3}$ at different time delays after excitation at 400 and $495 \mathrm{~nm}$.
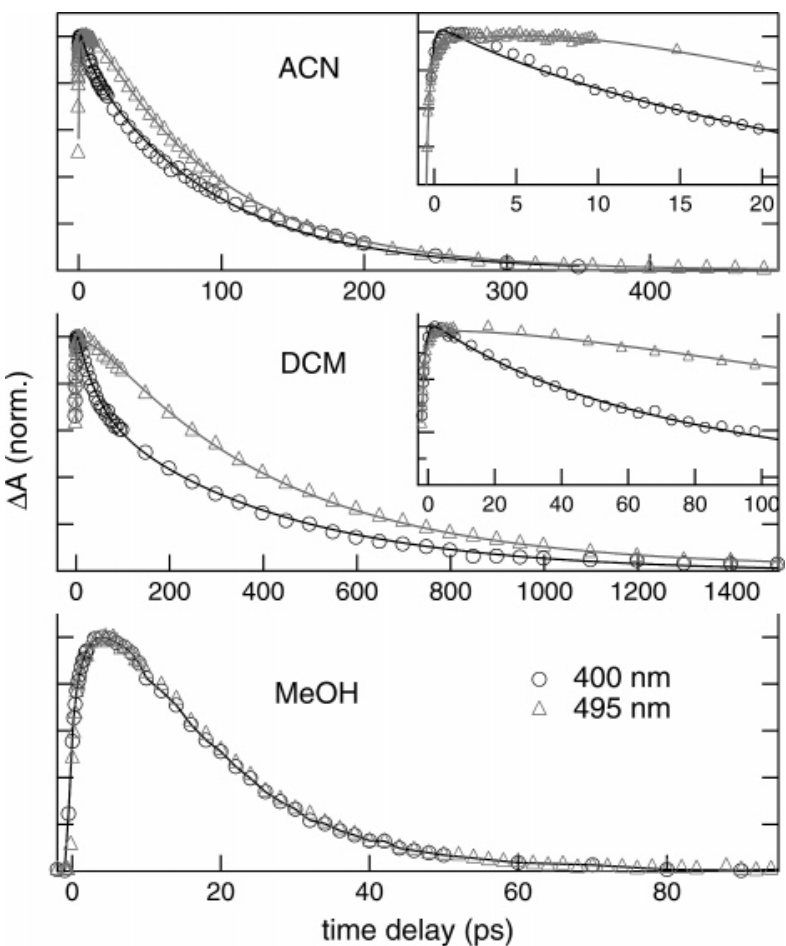

Figure 6. Time profiles of the TA intensity at the positive band maximum upon excitation at 400 and $495 \mathrm{~nm}$ and the best global fit.

the experiment, and no new band that could be ascribed to the triplet state can be observed.

TA spectra of $\mathrm{NPe}$ in $\mathrm{CHCl}_{3}, \mathrm{DCM}, \mathrm{MeOH}, \mathrm{ACN}$, and NMF have also been recorded upon $495 \mathrm{~nm}$ excitation. The spectra are qualitatively similar to those measured upon $400 \mathrm{~nm}$ excitation. However, a few differences can be observed at early time in $\mathrm{CHCl}_{3}$ and DCM (see Figure 5). First, the initial position of the band maximum is red-shifted by 3-4 $\mathrm{nm}$ compared to $400 \mathrm{~nm}$ excitation, and second, the increase of the TA intensity that occurs in parallel to the blue shift is about twice as large upon $495 \mathrm{~nm}$ excitation. On the other hand, the time evolution of the shift and the final position of the band maximum are essentially the same. In ACN, no early evolution of the TA spectrum can be observed, as also found with $400 \mathrm{~nm}$ excitation. Finally, in $\mathrm{MeOH}$ and NMF, no significant difference in the TA spectrum can be seen. Both the magnitude of the blue shift and the amplitude of the initial rise are the same upon blue and red side excitations.

Figure 6 shows the time evolution of the TA intensity at a wavelength corresponding to the maximum of the positive band after the initial blue shift measured in several solvents. In $\mathrm{MeOH}$ and in NMF, both time profiles are independent of the excitation 
TABLE 3: Best-Fit Parameters Obtained from Analysis of the Time Profile of the TA Intensity at the Positive Band Maximum

\begin{tabular}{llrrlrrl}
\hline solvent & $\lambda_{\text {ex }}(\mathrm{nm})$ & $\tau_{1}(\mathrm{ps})$ & \multicolumn{1}{c}{$A_{1}$} & $\tau_{2}(\mathrm{ps})$ & $\mathrm{A}_{2}$ & $\tau_{3}(\mathrm{ps})$ & $A_{3}$ \\
\hline $\mathrm{CHX}$ & 400 & 25.6 & -0.19 & $1700^{a}$ & 1.0 & & \\
$\mathrm{CHCl}_{3}$ & 400 & 3.1 & -.69 & 38 & 0.26 & 194 & 0.74 \\
& 495 & & -.60 & & -0.25 & & 1.0 \\
$\mathrm{DCM}$ & 400 & 0.95 & -0.44 & 48 & 0.34 & 409 & 0.66 \\
& 495 & & -0.34 & & -0.11 & & 1.0 \\
$\mathrm{MeOH}$ & 400,495 & 2.6 & -0.44 & 16.1 & 1.0 & & \\
$\mathrm{MeOD}$ & 400 & 2.9 & -0.40 & 21.5 & 1.0 & & \\
$\mathrm{ACN}$ & 400 & 0.17 & -0.13 & 7.9 & 0.11 & 90 & 0.89 \\
& 495 & & -0.34 & & -0.17 & & 1.0 \\
$\mathrm{DMSO}$ & 400 & 2.4 & -0.7 & 97 & 0.36 & 674 & 0.62 \\
$\mathrm{NMF}$ & 400,495 & 1.6 & -0.57 & 34.5 & 1.0 & & \\
$\mathrm{IL}$ & 400 & 1.3 & -0.13 & 17 & -0.33 & 567 & 1.0
\end{tabular}

${ }^{a}$ Obtained by TCSPC.

wavelength and can be perfectly reproduced using the sum of two exponential functions, a rising one with a time constant on the order of a few picoseconds, which reflects both the blue shift and the increase of the positive TA band intensity, and a decaying one with a time constant around $16 \mathrm{ps}$ in $\mathrm{MeOH}$ and 34 ps in NMF (see Table 3).

On the other hand, in $\mathrm{DCM}, \mathrm{ACN}$, and $\mathrm{CHCl}_{3}$, the decay of the positive TA band is markedly faster upon $400 \mathrm{~nm}$ excitation. A global analysis of these kinetics using the sum of three exponential functions results in a good fit to the data, as shown by the continuous lines in Figure 6, the best-fit parameters being listed in Table 3. At both pump wavelengths, the TA intensity exhibits first a prompt rise followed by a slower rise, with a time constant ranging from 0.17 to $3.1 \mathrm{ps}$. The amplitude associated with the second time constant is negative upon 400 $\mathrm{nm}$ excitation and positive at $495 \mathrm{~nm}$ pumping, corresponding to a decay and a rise of the signal, respectively. Finally, the third time constant is related to a positive amplitude for both pump wavelengths and reflects the decay of the excited-state population.

The time profiles of the TA at the band maximum (after blue shift) in the other solvents upon $400 \mathrm{~nm}$ excitation were also analyzed with the sum of three exponential functions, and the resulting parameters are listed in Table 3 .

The rising component associated with $\tau_{1}$ is eliminated when considering the time evolution of the integral of the TA intensity over of the whole spectrum instead of the positive band maximum after the initial blue shift. This clearly indicates that the fast rising component arises totally from the initial spectral dynamics due to solvation. Apart from this initial rise, the same differences between the 400 and $495 \mathrm{~nm}$ excitations are observed when considering the integrated spectrum and the intensity of the positive TA band maximum.

Finally, TA measurements have also been performed in perdeuterated methanol, MeOD. While the time profile of the TA intensity at the positive band maximum is qualitatively very similar to that in $\mathrm{MeOH}$, an increase of the time constant associated with the population decay from 16.1 to 21.5 ps can be observed.

Quenching by TCNE. The nitro substituent can be expected to decrease the electronic density on the Pe core, which should thus have some cationic character in the $S_{1}$ state. In order to see the influence of this effect on the excited-state spectrum of $\mathrm{NPe}$, the radical cation $\mathrm{NPe}^{\bullet+}$ was generated by ET quenching with TCNE in ACN. Figure 7A shows TA spectra recorded at several time delays after $400 \mathrm{~nm}$ excitation of NPe in the presence of $0.9 \mathrm{M}$ TCNE. At first sight, the TA spectra do not differ from those measured without TCNE. However a few
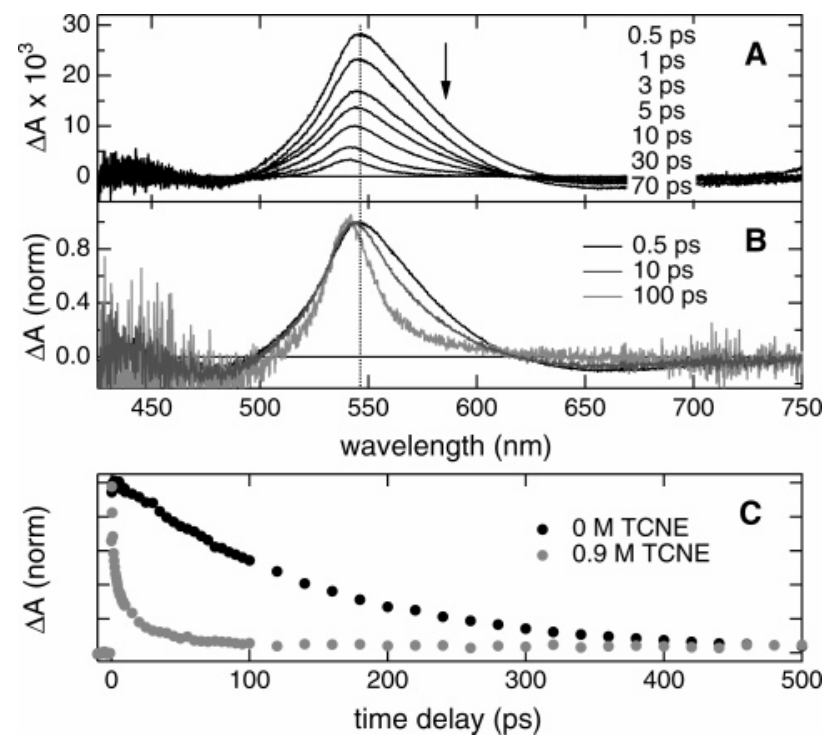

Figure 7. (A) TA spectra recorded at different time delays after 400 $\mathrm{nm}$ excitation of NPe with $0.9 \mathrm{M}$ TCNE in ACN; (B) comparison of intensity-normalized TA spectra; (C) time profiles of the TA intensity at $545 \mathrm{~nm}$ with and without TCNE.

significant differences can be noticed. First, a blue shift and a narrowing of the positive TA band occur in parallel to its decay, a comparison of intensity-normalized TA spectra being shown in Figure 7B. Second, the time dependence of the TA spectrum differs totally in the presence of TCNE, as shown Figure 7C. At wavelengths corresponding to the positive band, the TA loses more than $90 \%$ of its initial intensity during the first 40 ps and then remains almost constant up to $2 \mathrm{~ns}$. On the other hand, the time profile of the stimulated emission intensity only exhibits the fast decay component and has totally vanished after $40 \mathrm{ps}$. This fast decay component can thus be ascribed to the quenching of NPe* $\left(\mathrm{S}_{1}\right)$ upon ET by TCNE, and the TA band after 50 ps can be assigned to $\mathrm{NPe}^{\cdot+}$. Its absorption spectrum is similar to that of $\operatorname{NPe}^{*}\left(\mathrm{~S}_{1}\right)$ but is narrower and $7 \mathrm{~nm}\left(240 \mathrm{~cm}^{-1}\right)$ blueshifted, explaining the time evolution of the TA spectra of NPe with TCNE. The very weak positive TA band below $480 \mathrm{~nm}$ is possibly due to $\mathrm{TCNE}^{\bullet-}$, which is known to absorb in this region. ${ }^{42}$ The ET quenching of $\mathrm{Pe} *\left(\mathrm{~S}_{1}\right)$ by TCNE has been shown to be rather complex, ${ }^{19,43,44}$ and to result in the formation of both strongly coupled ion pairs, undergoing ultrafast charge recombination, and longer-lived weakly coupled ion pairs. The longer-lived $\mathrm{NPe}^{\bullet+}$ band observed here most probably corresponds to such weakly coupled ion pairs, which can then dissociate into free ions. On the other hand, strongly coupled ion pairs are most certainly generated as well but decay on a similar time scale as that of quenching and thus do not contribute significantly to the TA spectra. However, a detailed analysis of the quenching mechanism goes beyond the main aim of the present study.

Comparison with NPy. To confirm that the ultrashort fluorescence lifetime of smaller nitroaromatic hydrocarbons is indeed due to ISC, ${ }^{11}$ TA measurements have been performed with nitropyrene (NPy) in ACN. At early time, the TA spectrum (Figure 8A) consists of a relatively narrow and intense band with a maximum at $480 \mathrm{~nm}$ and a weaker and broad band culminating around $670 \mathrm{~nm}$. The intensity of both bands decreases with time, while a new feature between 530 and 620 $\mathrm{nm}$ increases. A global analysis of these TA spectra recorded after $600 \mathrm{fs}$ using eq 1 resulted in a good fit with a time constant of $3.8 \mathrm{ps}$ and with the spectra of the amplitude factors, $A_{1}$ and $A_{2}$, shown in Figure 8B. This 3.8 ps time constant agrees very 


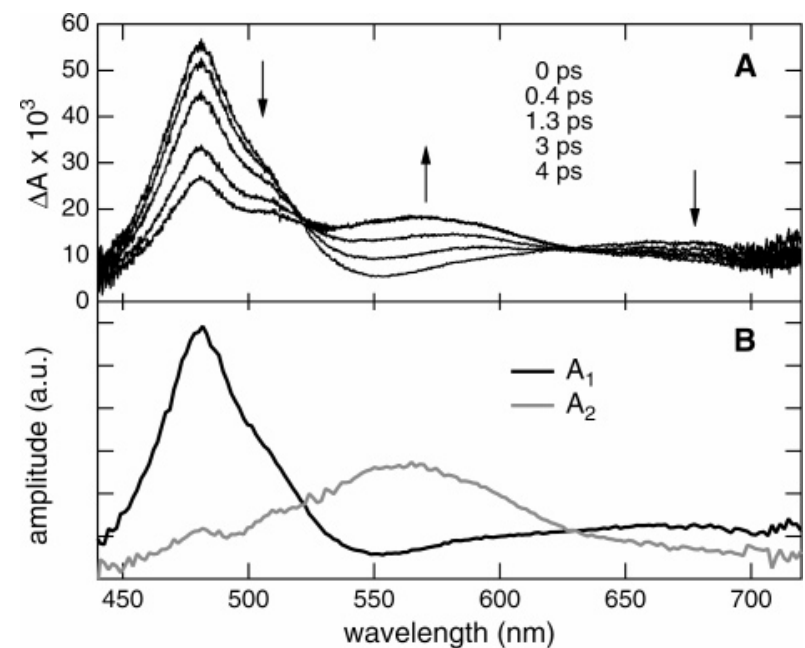

Figure 8. (A) TA spectra recorded at different time delays after 400 $\mathrm{nm}$ excitation of NPy in ACN; (B) spectral dependence of the amplitude factors obtained from global analysis (see text).

well with the larger time constant observed in the fluorescence decay of $\mathrm{NPy}$ in $\mathrm{MeOH} .{ }^{11}$ A smaller time constant ranging between 500 and $800 \mathrm{fs}$, depending on the fluorescence wavelength, was also detected and ascribed to fast intramolecular rearrangement. Such a process could be responsible for the initial rising component of the TA signal, but this effect was not investigated further. Despite this, the TA measurements clearly confirm the very short $\mathrm{S}_{1}$ lifetime of NPy upon ISC. The spectrum of $A_{1}$ can be ascribed to $\mathrm{S}_{1}-\mathrm{S}_{n}$ absorption with some possible contribution of stimulated emission between 500 and $570 \mathrm{~nm}$, while the spectrum of $A_{2}$ can be assigned to NPy*$\left(\mathrm{T}_{1}\right)$.

\section{Discussion}

Nature of $\mathbf{N P e}^{*}\left(\mathbf{S}_{\mathbf{1}}\right)$. TDDFT calculations of NPe in the gas phase predict the first electronic transition from the ground state at $483 \mathrm{~nm}$, in good agreement with the absorption maximum at $460 \mathrm{~nm}$ measured in CHX. According to these calculations, the $\mathrm{S}_{1}$ state is associated with a one-electron HOMO-LUMO transition. Figure 9A shows that the electronic density at the nitro group is substantially larger in the LUMO than that in the HOMO, pointing to a substantial charge-transfer (CT) character of the $\mathrm{S}_{0}-\mathrm{S}_{1}$ transition. This is in perfect agreement with the significant increase of the electric dipole moment deduced from the solvatochromism of the steady-state spectra. The decrease of electronic density on the Pe core also explains the similarity between the spectra of $\mathrm{NPe} *\left(\mathrm{~S}_{1}\right)$ and $\mathrm{NPe}^{\bullet+}$ measured in $\mathrm{ACN}$, the latter being almost identical to that of $\mathrm{Pe}^{\bullet+}$. The spectra of $\mathrm{NPe}^{*}\left(\mathrm{~S}_{1}\right)$ in solvents of similar polarity, like $\mathrm{MeOH}$ and DMSO, strongly resemble that in $\mathrm{ACN}$, once solvent relaxation is complete. In $\mathrm{CHX}$ on the other hand, the spectrum of NPe*$\left(\mathrm{S}_{1}\right)$ differs considerably from those of $\mathrm{NPe}^{{ }^{+}}$and $\mathrm{Pe} *\left(\mathrm{~S}_{1}\right)$, the latter peaking at $700 \mathrm{~nm}$. The difference between the spectrum in $\mathrm{CHX}$ and those in polar solvents could be interpreted as an increase of the CT character of the excited state with solvent polarity. This might be due to the polarization exerted by the solvent reaction field. Such a solvation-induced polarization can also explain the blue shift of the positive TA band observed at early time in polar solvents. An alternative explanation for the solvent dependence of the TA spectrum is that the CT character of the final state of the $\mathrm{S}_{1}-\mathrm{S}_{n}$ transition is small. This would also lead to an increase of the $S_{1}-S_{n}$ transition energy with increasing solvent polarity.

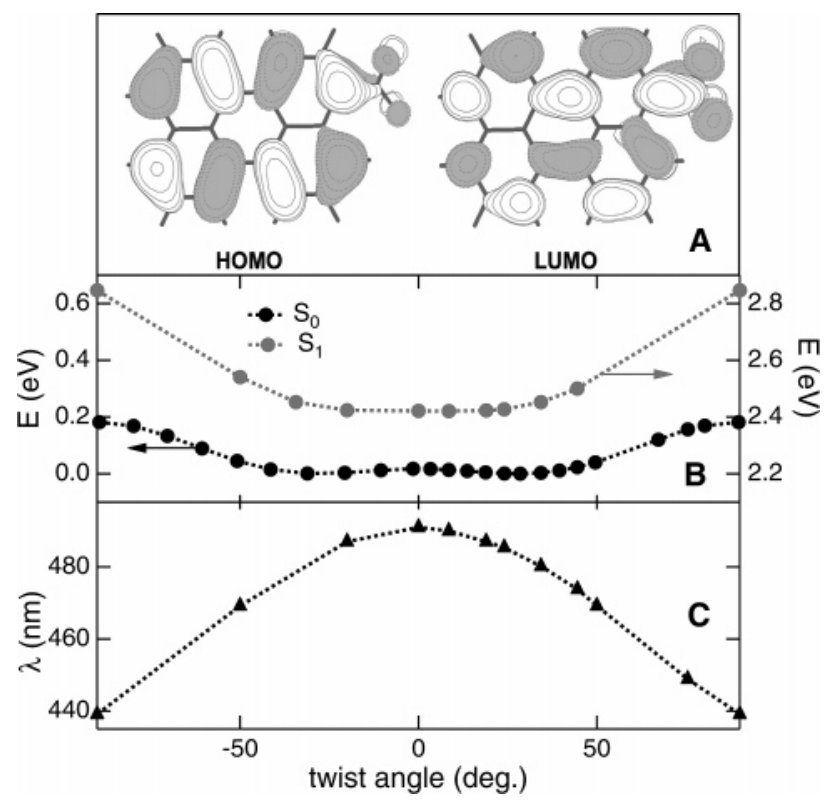

Figure 9. (A) Frontier molecular orbitals of NPe drawn at the $0.02 \mathrm{au}$ level; (B) calculated relative energy of NPe in the $S_{0}$ and $S_{1}$ states and (C) the $\mathrm{S}_{0}-\mathrm{S}_{1}$ transition energy as a function of the $\mathrm{NO}_{2}$ twist angle (the dotted lines are only guides for the eyes).

DFT calculations indicate that in the equilibrium ground-state geometry, the nitro group is planar and makes a twist angle of $\pm 27^{\circ}$ relative to the aromatic plane. However, the energy minimum along the torsional coordinate of the nitro group is very shallow between -50 and $50^{\circ}$, as illustrated in Figure 9B. As a consequence, a distribution of ground-state geometries with different twist angles can be expected at room temperature. Similar conclusions have been drawn for nitrobenzene and p-nitroaniline. ${ }^{14,16}$

On the other hand, the gas-phase equilibrium geometry of $\mathrm{NPe} *\left(\mathrm{~S}_{1}\right)$ is predicted to be planar (Figure $9 \mathrm{~B}$ ). Moreover, the potential along the torsional coordinate is not as shallow as that for the ground state. Figure $9 \mathrm{C}$ shows how the $\mathrm{S}_{0}-\mathrm{S}_{1}$ transition energy depends on the $\mathrm{NO}_{2}$ twist angle. It thus appears that light at a short wavelength should predominantly interact with molecules with a large twist angle, while longer wavelength excitation should rather photoselect planar molecules. It should be noted that the situation in solution might differ substantially from that calculated in the gas phase. Nevertheless, these calculations offer a good basis for a qualitative discussion of the pump wavelength dependence of NPe excited-state dynamics. Moreover, they give a reasonable explanation for the absence of a mirror image relationship between the absorption and the fluorescence spectra observed in nonprotic solvents. Indeed, a fluorescence band narrower than the corresponding absorption band can be explained by a reduction of the torsional disorder in the excited state, in agreement with the less shallow potential predicted by calculations. Similar effects have also been reported with polyphenylenethynylenes. ${ }^{45,46}$ The fact that the mirror image relationship exists in protic solvents can be explained by the formation of $\mathrm{H}$-bonds with the solvents that restrict the twisting motion of the nitro group and thus reduces torsional disorder.

Excited-State Dynamics. The excited-state dynamics of NPe differs completely from that of the smaller nitroaromatic hydrocarbons, which undergo ultrafast ISC to the triplet state, ${ }^{11}$ as clearly confirmed here with NPy. In the case of NPe, ISC is only observed in CHX and, as the triplet quantum yield $\Phi_{\mathrm{T}} \leq$ $1-\Phi_{\mathrm{fl}}$, takes place with a time constant larger than $2 \mathrm{~ns}$. The 
origin of the ultrafast ISC in smaller nitroaromatic compounds lies in the existence of a triplet state of $n \pi^{*}$ character associated with the nitro group with similar energy as the $S_{1} \pi \pi^{*}$ state. ${ }^{12}$ This warrants very fast ISC due to a large spin-orbit coupling, in agreement with the El-Sayed rules. ${ }^{47}$ Our results evidence that such a process is not operative with NPe. A probable reason is that the increased size of the aromatic system of NPe leads to a substantial decrease of the energy of the $\pi \pi^{*} \mathrm{~S}_{1}$ state relative to those of the triplet $n \pi^{*}$ states associated with the nitro group, which thus do not depend as much on the size of the aromatic system.

The present results show that the excited-state dynamics of $\mathrm{NPe}$ is strongly solvent-dependent. In nonpolar solvents, NPe*$\left(\mathrm{S}_{1}\right)$ is rather long-lived and decays mainly by ISC and fluorescence. However, as the triplet quantum yield was not determined, the occurrence of IC cannot be ruled out.

The shortest $\mathrm{S}_{1}$ state lifetimes have been measured in protic solvents. The substantial isotopic effect, $\tau_{\mathrm{D}} / \tau_{\mathrm{H}} \sim 1.4$, observed in methanol, strongly points to the occurrence of reversible intermolecular proton transfer. ${ }^{48,49}$ Such a process agrees with the increase of electron density at the nitro group in the $S_{1}$ state. Moreover, the occurrence of the $\mathrm{H}$-bonding interaction was already suspected to be at the origin of the mirror image symmetry between absorption and emission spectra in protic solvents. The absence of an excitation wavelength dependence on the excited-state dynamics in $\mathrm{MeOH}$ and $\mathrm{NMF}$ is also coherent with an essentially homogeneous $\mathrm{S}_{0}-\mathrm{S}_{1}$ absorption band in protic solvents. Both the H-bonding interaction already in the ground state and a higher electron density in the $S_{1}$ state warrant ultrafast proton transfer. The fact that the product of such a process cannot be observed in the TA spectra could be explained by a very short lifetime because of ultrafast backward transfer, regenerating NPe in the electronic ground state.

The interpretation of the results obtained in polar and nonprotic solvents is less straightforward. The data listed in Table 3 show that the main decay time of NPe*( $\left.\mathrm{S}_{1}\right), \tau_{3}$, varies from $90 \mathrm{ps}$ in ACN to $567 \mathrm{ps}$ in IL. If only the most polar solvents are considered, a correlation between $\tau_{3}$ and solvent viscosity seems to exist. This points to a nonradiative deactivation of the excited state via an intramolecular mode with large amplitude motion. ${ }^{50,51}$ On the other hand, the decay time $\tau_{3}$ is relatively large in the weakly polar solvents, $\mathrm{CHCl}_{3}$ and DCM, although their viscosity is only slightly above that of ACN. Such a dependence of the excited-state lifetime on both the solvent viscosity and polarity has already been observed in processes involving large-amplitude motion, with a barrier decreasing with solvent polarity. ${ }^{52,53}$ Such a situation can be reasonably envisaged here with both the low-frequency twisting and wagging modes of the nitro group and considering the CT character of the $S_{1}$ state. Thus, we propose that in nonprotic and polar solvents, $\mathrm{NPe} *\left(\mathrm{~S}_{1}\right)$ decays essentially nonradiatively via a lowfrequency coordinate associated with the nitro group. This process is favored in more polar solvents because of the reduction of the $S_{1}-S_{0}$ energy gap though dipole-dipole interaction. In CHX, this process is so slow that ISC becomes competitive.

The wavelength dependence of the excited-state dynamics observed in nonprotic solvents can be explained by a scheme where the excited subpopulations, initially created at distinct Franck-Condon regions of the excited-state potential, evolve toward a common region, which corresponds to the thermally equilibrated excited state. Once there, both populations behave similarly, namely, they decay with the time constant $\tau_{3}$. The evolution from the Franck-Condon regions involves relaxation along the solvation coordinate, responsible for the blue shift of the TA band and associated with the time constant $\tau_{1}$, as well as motion along intramolecular coordinates of the nitro group and related to the time constant $\tau_{2}$. Two effects could account for the opposite signs of the amplitude of the $\tau_{2}$ component upon 400 or $495 \mathrm{~nm}$ excitation and the excitation wavelength dependence of the fluorescence quantum yield.

1) Nonequilibrium IC. Part of the excited subpopulation generated at $400 \mathrm{~nm}$ could decay to the ground state already during relaxation. This would imply that the probability of IC is larger near the $400 \mathrm{~nm}$ Franck-Condon region than that around the equilibrium region. This is not the case for the subpopulation created upon $495 \mathrm{~nm}$ pumping as the amplitude associated with the time constant $\tau_{2}$ is negative. This suggests that the probability for IC directly from the $495 \mathrm{~nm}$ FranckCondon region is essentially zero and that, in this case, IC essentially takes place from equilibrium. The time profile of the excited-state population in such a situation remains rather flat at early time before the onset of the decay. Such a profile can either be reproduced with the sum of rising and decaying exponentials or with a compressed exponential function, as demonstrated recently for excited donor-acceptor complexes undergoing nonequilibrium charge recombination. ${ }^{54,55}$ The advantage of the multiexponential function is the possibility to perform global analysis with the dynamics measured upon 400 $\mathrm{nm}$ excitation. This hypothesis is supported by the analysis of the time profile of the TA intensity in the region corresponding to the bleach of the $\mathrm{S}_{0}-\mathrm{S}_{1}$ absorption, which shows a biphasic ground-state recovery dynamics with time constants similar to $\tau_{2}$ and $\tau_{3}$ upon $400 \mathrm{~nm}$ excitation. Unfortunately, this spectral region was not accessible upon $495 \mathrm{~nm}$ excitation because of the use of a cutoff filter to remove the scattered pump light.

2) Variation of Oscillator Strength. The oscillator strength for the $\mathrm{S}_{1}-\mathrm{S}_{n}$ transition could vary upon relaxation from the Franck-Condon regions toward equilibrium, decreasing after $400 \mathrm{~nm}$ excitation and increasing upon $495 \mathrm{~nm}$ pumping. However, such a variation alone cannot explain the dependence of the fluorescence quantum yield on the pump wavelength unless the oscillator strength for the $S_{1}-S_{0}$ fluorescence exhibits a similar variation upon relaxation. The stimulated emission in polar solvents was too weak to accurately detect a dependence of its time profile on the excitation wavelength. However, it should be noted that in $\mathrm{CHX}$, where stimulated emission is more intense, the 25 ps rising component detected in the positive TA band region was also observed in the stimulated emission region, that is, between 500 and $550 \mathrm{~nm}$. However, this component was clearly absent at shorter wavelengths, where the TA signal is due to the bleach of the $\mathrm{S}_{0}-\mathrm{S}_{1}$ absorption. This shows that this 25 ps component is not associated with population dynamics but could be due to a variation of the oscillator strength arising from structural changes of $\mathrm{NPe} *\left(\mathrm{~S}_{1}\right)$ upon relaxation. Moreover, quantum chemistry calculations on para-nitroaniline have revealed rather parallel variations of the oscillator strength of the $\mathrm{S}_{1}-\mathrm{S}_{n}$ and $\mathrm{S}_{1}-\mathrm{S}_{0}$ transitions with the $\mathrm{NO}_{2}$ twist angle. ${ }^{14}$

As a consequence, the observed excitation wavelength dependence is most probably due to a combination of both phenomena.

\section{Concluding Remarks}

This investigation shows that the photophysics of NPe differs considerably from that of smaller nitroaromatic hydrocarbons, which, as confirmed here with NPy, undergo ultrafast ISC to the $\mathrm{T}_{1}$ state. Indeed, ISC was only observed with NPe in nonpolar solvents. Instead, the main deactivation pathway of 
$\operatorname{NPe} *\left(\mathrm{~S}_{1}\right)$ is IC, with a mechanism and dynamics that depend strongly on the solvent. Another interesting feature of NPe is the wavelength dependence of its excited-state dynamics that is related to the existence of a distribution of ground-state geometries differing mainly by the torsion angle of the nitro group relative to the aromatic plane. Interestingly, such inhomogeneous dynamics is absent in protic solvents, most probably because of the formation of $\mathrm{H}$-bonds, which limit the free torsion of the nitro group.

Finally, it is interesting to note that although the CT character of $\mathrm{NPe} *\left(\mathrm{~S}_{1}\right)$ is far from being unity, its absorption spectrum in polar solvents is very similar to that of $\mathrm{NPe}^{\bullet+}$, which is itself almost identical to that of $\mathrm{Pe}^{\bullet+}$. This explains why it is so problematic to differentiate the absorption spectrum of $\mathrm{Pe}^{\bullet+}$ in ion pairs with that of the free $\mathrm{Pe}^{\bullet+}$. According to the present results, a difference would only be seen in the case of a very strongly coupled ion pair where the extent of CT character is substantially smaller than one. In this case, the absorption spectrum of the paired cation would be slightly red-shifted and broader than that of the free cation.

Acknowledgment. This work was supported by the Fonds National Suisse de la Recherche Scientifique through Project No. 200020-115942.

\section{References and Notes}

(1) Hurley, R.; Testa, A. C. J. Am. Chem. Soc. 1968, 90, 1949.

(2) Anderson, R. W.; Hochstrasser, R. M.; Lutz, H.; Scott, G. W. Chem Phys. Lett. 1974, 28, 153.

(3) Hamanoue, K.; Hirayama, S.; Nakayama, T.; Teranishi, H. J. Phys. Chem. 1980, 84, 2074.

(4) Thomsen, C. L.; Thogersen, J.; Keiding, S. R. J. Phys. Chem. A 1998, 102, 1062.

(5) Gurzadyan, G.; Goerner, H. Chem. Phys. Lett. 2000, 319, 164. 1159 .

(6) Capellos, C.; Porter, G. J. Chem. Soc., Faraday Trans. 2 1974, 70, 321.

Gorner, H.; Schulte-Frohlinde, D. Chem. Phys. Lett. 1986, 124

(8) Fournier, T.; Tavender, S. M.; Parker, A. W.; Scholes, G. D.; Phillips, D. J. Phys. Chem. A 1997, 101, 5320.

(9) Fawi, M.; Latif, A. E. J. Photochem. Photobiol., A 2001, 141, 241

(10) Goerner, H.; Doepp, D. J. Chem. Soc., Perkin Trans. 2002, 120

(11) Morales-Cueto, R.; Esquivelzeta-Rabell, M.; Saucedo-Zugazagoitia,

J.; Peon, J. J. Phys. Chem. A 2007, 111, 552.

(12) Zugazagoitia, J. S.; Almora-Diaz, C. X.; Peon, J. J. Phys. Chem. A 2008, 112,358 .

(13) Kovalenko, S. A.; Schanz, R.; Farztdinov, V. M.; Hennig, H.; Ernsting, N. P. Chem. Phys. Lett. 2000, 323, 312.

(14) Farztdinov, V. M.; Schanz, R.; Kovalenko, S. A.; Ernsting, N. P.

J. Phys. Chem. A 2000, 104, 11486.

(15) Takezaki, M.; Hirota, N.; Terazima, M. J. Phys. Chem. A 1997, $101,3443$.

(16) Takezaki, M.; Hirota, N.; Terazima, M.; Sato, H.; Nakajima, T.; Kato, S. J. Phys. Chem. A 1997, 101, 5190.
(17) Vauthey, E.; Suppan, P.; Haselbach, E.; Davidson, R. S. Helv. Chim. Acta 1986, 69, 430.

(18) Mataga, N.; Asahi, T.; Kanda, Y.; Okada, T.; Kakitani, T. Chem. Phys. 1988, 127, 249.

(19) Pagès, S.; Lang, B.; Vauthey, E. J. Phys. Chem. A 2004, 108, 549

(20) Pagès, S.; Lang, B.; Vauthey, E. J. Phys. Chem. A 2006, 110, 7547.

(21) Dewar, M. J. S.; Mole, T. J. Chem. Soc. 1956, 1441.

(22) Lewis, J. E.; Maroncelli, M. Chem. Phys. Lett. 1998, 282, 197.

(23) Fürstenberg, A.; Julliard, M. D.; Deligeorgiev, T. G.; Gadjev, N. I.; Vassilev, A. A.; Vauthey, E. J. Am. Chem. Soc. 2006, 128, 7661. 5361 .

(24) Duvanel, G.; Banerji, N.; Vauthey, E. J. Phys. Chem. A 2007, 111,

(25) Wilhelm, T.; Piel, J.; Riedle, E. Opt. Lett. 1997, 22, 1494.

(26) Perdew, J. P. Phys. Rev. B 1986, 33, 8822.

(27) Schäfer, A.; Horn, H.; Ahlrichs, R. J. Chem. Phys. 1992, 97, 2571.

(28) Bauernschmitt, R.; Ahlrichs, R. Chem. Phys. Lett. 1996, 256, 454.

(29) Ahlrichs, R.; Bär, M.; Häser, M. Chem. Phys. Lett. 1989, 162, 165.

(30) Häser, M.; Ahlrichs, R. J. Comput. Chem. 1989, 10, 104.

(31) Suppan, P. J. Photochem. Photobiol., A 1990, 50, 293.

(32) Suppan, P.; Ghoneim, N. Solvatochromism; The Royal Society of Chemistry: Cambridge, U.K., 1997.

(33) Lippert, E. Z. Naturforsch. 1955, 10, 541.

(34) Mataga, N.; Kaifu, Y.; Koizumi, M. Bull. Chem. Soc. Jpn. 1956 $29,465$.

(35) Angulo, G.; Grampp, G.; Rosspeintner, A. Spectrochim. Acta A 2006, 65, 727 .

(36) Horng, M. L.; Gardecki, J. A.; Papazyan, A.; Maroncelli, M. J. Phys. Chem. 1995, 99, 17311.

(37) Stratt, R. M.; Maroncelli, M. J. Phys. Chem. 1996, 100, 12981.

(38) Rosenthal, S. J.; Xie, X.; Du, M.; Fleming, G. R. J. Chem. Phys. 1991, 95, 4715 .

(39) Karmakar, R.; Samanta, A. J. Phys. Chem. A 2002, 106, 4447.

(40) Lang, B.; Angulo, G.; Vauthey, E. J. Phys. Chem. A 2006, 110, 7028

(41) Arzhantsev, S.; Jin, H.; Baker, G. A.; Maroncelli, M. J. Phys. Chem. B 2007, 111, 4978 .

(42) Shida, T. Electronic Absorption Spectra of Radical Ions; Elsevier: Amsterdam, The Netherlands, 1988; Vol. Physical Sciences data 34.

(43) Gladkikh, V.; Burshtein, A. I.; Angulo, G.; Pagès, S.; Lang, B.; Vauthey, E. J. Phys. Chem. A 2004, 108, 6667.

(44) Gladkikh, V. S.; Burshtein, A. I.; Feskov, S. V.; Ivanov, A. I.; Vauthey, E. J. Chem. Phys. 2005, 123, 244510.

(45) Sluch, M. I.; Godt, A.; Bunz, U. H. F.; Berg, M. A. J. Am. Chem. Soc. 2001, 123, 6447.

(46) Duvanel, G.; Grilj, J.; Schuwey, A.; Gossauer, A.; Vauthey, E. Photochem. Photobiol. Sci. 2007, 6, 956.

(47) Lower, S. K.; El-Sayed, M. A. Chem. Rev. 1966, 66, 199. 477 .

(49) Agmon, N. J. Phys. Chem. A 2005, 109, 13.

(50) Velsko, S. P.; Fleming, G. R. Chem. Phys. 1982, 65, 59.

(51) Bagchi, B.; Fleming, G. R. J. Phys. Chem. 1990, 94, 9.

(52) Hicks, J. M.; Vandersall, M. T.; Sitzmann, E. V.; Eisenthal, K. B. Chem. Phys. Lett. 1987, 135, 413.

(53) Grabowski, Z. R.; Rotkiewicz, K.; Rettig, W. Chem. Rev. 2003, $103,3899$.

(54) Fedunov, R. G.; Feskov, S. V.; Ivanov, A. I.; Nicolet, O.; Pagès, S.; Vauthey, E. J. Chem. Phys. 2004, 121, 3643.

(55) Nicolet, O.; Banerji, N.; Pagès, S.; Vauthey, E. J. Phys. Chem. A 2005, 109,8236 . 